\title{
Neuroependymal denudation is still in progress in full-term human fetal spina bifida aperta
}

\author{
Deborah Sival ${ }^{*}$, Montserrat Guerra ${ }^{2}$, Wilfred den Dunnen ${ }^{3}$, Frederico Bátiz², Genaro Alvial ${ }^{2}$, Esteban Rodríguez ${ }^{2}$ \\ From 54th Annual Meeting of the Society for Research into Hydrocephalus and Spina Bifida \\ Vancouver, Canada. 7-10 July 2010
}

\section{Background}

Background: In human spina bifida aperta (SBA), cerebral pathogenesis (hydrocephalus, Sylvian aqueduct (SA) stenosis and heterotopias) is poorly understood. In animal models (such as hyh mutant mice) the loss of the ventricular lining (ependymal denudation) causes SA stenosis and hydrocephalus. In these animals, ependymal denudation is ascribed to an alteration in junction proteins. Analogous to studies in laboratory animals, we aimed to investigate ependymal denudation in human fetal SBA.

\section{Materials and methods}

Sections through SA of five SBA and five control fetuses (median gestational ages 37 and 40 weeks, respectively) were immunostained for markers of ependyma (caveolin1, $\beta$ IV-tubulin, S100), blood vessels (Glut-1), astrocytes (GFAP) and junction proteins (N-cadherin, connexin-43, neural cell adhesion molecule (NCAM)).

\section{Results}

In all five control fetuses, ependymal denudation was absent. In all five SBA fetuses, different stages of ependymal denudation were concurrently observed, consisting of: (I) intact ependyma/neuroepithelium; (II) imminent ependymal denudation (with abnormal subcellular location of junction proteins in cytoplasm instead of at the plasma membrane); (III) ongoing ependymal denudation (with protrusion of neuropil into SA, formation of rosettes and macrophage invasion); and (IV) completed ependymal denudation (with astroglial reaction).

\section{Conclusions}

In full-term SBA fetuses, intra-individual concurrence of imminent, ongoing and completed ependymal denudation implicates that ependymal denudation would continue after birth. At the areas associated with imminent ependymal denudation, the abnormal expression of junction proteins suggests that abnormal formation of gap and adherent junctions precedes defective ependymal coupling, desynchronized ciliary beating, ependymal denudation and hydrocephalus.

Author details

${ }^{1}$ Department of Pediatric Neurology University Medical Center Groningen, University of Groningen, Beatrix Children's Hospital, Hanzeplein 1, PO Box 30001, 9700RB, Groningen, The Netherlands. 'Instituto de Anatomía, Histología y Patología, Facultad de Medicina, Universidad Austral de Chile, Valdivia, Chile. ${ }^{3}$ Department of Pathology and Medical Biology, University Medical Center Groningen, University of Groningen, Beatrix Children's Hospital, Hanzeplein 1, PO Box 30001, 9700RB, Groningen, The Netherlands.

Published: 15 December 2010

doi:10.1186/1743-8454-7-S1-S19

Cite this article as: Sival et al:: Neuroependymal denudation is still in progress in full-term human fetal spina bifida aperta. Cerebrospinal Fluid Research 2010 7(Suppl 1):S19.

\footnotetext{
* Correspondence: d.a.sival@bkk.umcg.nl

'Department of Pediatric Neurology University Medical Center Groningen, University of Groningen, Beatrix Children's Hospital, Hanzeplein 1, PO Box 30001, 9700RB, Groningen, The Netherlands

Full list of author information is available at the end of the article
} 